\title{
Investigation of Inhibition Potential Effect of Organic Compound for the Corrosivity of Phosphoric Acid on Aluminium
}

\author{
Musa Husaini and Muhammad Bashir Ibrahim \\ Department of Pure and Industrial Chemistry, Faculty of Physical Sciences, Bayero University Kano (BUK), \\ P.M.B. 3011, Kano, Nigeria
}

Received: 24 September 2019; Accepted: 15 October 2019; Published: 08 February 2020

\begin{abstract}
The organic compound used as corrosion inhibitor was benzaldehyde. The use of organic compounds as corrosion inhibitors is of potential interest due to their abundant availability, cost effectiveness, and environmental acceptability. However, this study investigates the inhibition effect of this organic compound (benzaldehyde) on the corrosion of aluminium in phosphoric acid solution. Experiments were performed by varying the temperature and concentration of the compound under study. The results obtained from the study shows that the organic compound (benzaldehyde) is a potential inhibitor for the corrosion of aluminium in phosphoric acid solution. The inhibition efficiency was found to increases progressively as the concentration of the inhibitor increases but decreases when the temperature rises. Activation energy values for the corrosion process was found to be $32.61 \mathrm{~kJ} / \mathrm{mol}$ in uninhibited phosphoric acid solution which increased to $49.53 \mathrm{~kJ} / \mathrm{mol}$ in the presence $0.1 \mathrm{M}$ concentration of the inhibitor. The values of the rate constant and half live were $9.12 \times$ 10-3 hr-1 and $75.94 \mathrm{hr}$ in uninhibited phosphoric acid solution which changed to 4.56× 10-3 and $151.80 \mathrm{hr}$ in the presence of $0.1 \mathrm{M}$ inhibitor concentration. Kinetics of the reaction in uninhibited and in the presence of the inhibitor revealed that the process follows a first order reaction. The evaluated enthalpy $(\Delta \mathrm{H})$ gave positive values which indicate that the heat of adsorption process on the surface of aluminum was endothermic. The negative values of entropy $(\Delta \mathrm{S})$ signified that the activated complex at the rate determining step is an association, not dissociation. Evaluated values of free energy of adsorption ( $\Delta$ Gads) were all negative, implying spontaneity of the process and were around and less than $-20 \mathrm{~kJ} / \mathrm{mol}$, indicative of physisorption of the adsorption process.
\end{abstract}

Index Terms: Adsorption, aluminium, benzaldehyde, corrosion, FT-IR, Kinetic parameters and Thermodynamic parameters.

(C) 2020 Published by MECS Publisher. Selection and/or peer review under responsibility of the Research Association of Mode rn Education and Computer Science

* Corresponding author.

E-mail address: 


\section{Introduction}

Electrical Aluminium and its alloys have a wide variety of technological applications due to the balanced combination of chemical, mechanical, and physical properties like high ductility, stability for surface treatment, thermal and electrical conductivity, recycling ability and low density. In aqueous medium aluminium exhibits passive behavior as a result of formation of the strong passive oxide film on its surface. The passivating adhesive surface is there to protect the aluminium but due to its amphoteric nature cannot prevent corrosion of the aluminium by acids and bases as it dissolves readily on exposure to strong acidic and alkaline environment [1]. Phosphoric acid and other acids especially at high temperature, present a huge threat to the aluminium and as a major cause of corrosion. Phosphoric acid is highly strong corrosive mineral acid with the molecular formula H3PO4. Phosphoric acid is largely used for industrial acid cleaning and electro polishing of aluminium, it's therefore necessary to find a suitable way to reduce the aluminium corrosion in phosphoric acid [2]. Several ways has been used to reduce this effect such as cathodic protection and coating method, but the use of inhibitors for the prevention of corrosion of aluminium in contact with aggressive media is the most popular, practical and efficient method. Attention has been increased as a result of corrosion inhibitors for sulfuric acid, hydrochloric acid and phosphoric acid due to their extended applications [3]. The protection of metals against corrosion by phosphoric acid became the subject of much study since it has been used in so many industrial application especially in the production of fertilizer $[4,5]$. Generally, the heterogeneous organic compounds with electron density on their heteroatoms like $\mathrm{O}, \mathrm{N}$, S, etc. have the ability to resist corrosion. These heteroatoms served as the active center for the adsorption process on metal surfaces [6]. Additionally, compounds that have electronegative groups, $\pi$-bonds, conjugated double or triple bonds in their structures, usually exhibit good inhibitive properties. They supply electrons from the electron-rich site to the metals surface which they are effectively adsorbed [7]. In the present research work the effect of benzaldehyde as corrosion inhibitor for aluminum in $1.4 \mathrm{M}$ phosphoric acid solution is investigated. This study is aimed at investigating the inhibition potential effect of organic compound for the corrosive action of phosphoric acid on aluminium. The objectives of the study through which the aim was achieved are; study of the effect of inhibitor and temperature on the corrosion rate, study of the effect of inhibitor and temperature on inhibition efficiency and study of thermodynamic, kinetic and adsorptive properties of the organic compound (benzaldehyde) for the corrosive action of phosphoric acid on aluminium. Several method for measuring the corrosion rate of metallic substances in acidic solution has been reported in the literatures. But the weight loss method was use in the current research in measuring and monitoring the corrosion rate of aluminium in phosphoric acid solution.

\subsection{Significance of the Research}

The use of the studied organic compound (benzaldehyde) as corrosion inhibitor will become important as its relatively cheap, friendly, non-toxic and commercially available. Therefore, the studied compound can compete with eco-friendly inhibitors. In general this research provided a knowledge in which this investigated organic compound will serve as corrosion inhibitor in both domestic and industrial applications.

\subsection{Literature Review}

Many organic compounds such as aliphatic, aromatic and heterocyclic compound have been reported as corrosion inhibitors of aluminium in different corrosive medium [8, 9]. This is because these organic compounds contain hetero atoms which can easily protonate in acidic medium to give excellent inhibitory action on the corrosion of metallic substances [10]. 
The effect of Triethylenetetramine (TETA] and 2-(2-aminoethylamino) ethanol [AEAE] as corrosion inhibitors for N80 steel in 15\% $\mathrm{HCl}$ solution was studied by polarization, AC impedance (EIS) and weight loss measurements by Yadav et al., [11]. It was found that both the inhibitors were effective inhibitors and their inhibition efficiency was significantly increased with increasing concentration of inhibitors. Polarization studies revealed that the studied inhibitors represent mixed-type inhibitors. AC impedance studies revealed that charge transfer resistance increases and double layer capacitance decreases in presence of inhibitors. Adsorption of inhibitors at the surface of N80 steel was found to obey Langmuir isotherm.

Aniline was studied as a corrosion inhibitor for aluminum in trichloroacetic acid (TCA) solution by Desai [12]. The inhibition efficiency depended on the concentration of acid and the inhibitor. Inhibition efficiency of TCA solutions increased with aniline concentration, but decreased with rise in temperature, this corresponded to surface coverage of the metal by the inhibitor. The degrees of surface coverage, $\theta$, were calculated and found to increase with the inhibitor concentration. The results show also that, the inhibitor were adsorbed on the aluminum surface according to Langmuir adsorption isotherm. Polarization study reveals that the inhibitors function as a little anodic, but significant cathodic inhibitor.

Arvnabh et al., [13] investigated the the corrosion inhibition of aluminium alloys of grade 1060, 1100 and 3003 in trichloroacetic acid by using conductivity and potentiostatic polarization in different inhibitors of diamine such as ethyl amino ethylamine, di-methyl amino ethylamine, 1:3 di-amino propane, tetra methyl ethylene diamine. Cathodic and anodic polarization curves are showing diamines are acting as mixed inhibitors in the case of alloy of 3003 grade.

The inhibition effect of 1-(3-Nitrobenzylidene) Thiosemicarbazide (A) on the corrosion of mild steel in $0.5 \mathrm{M}$ sulphuric acid have been investigated at room temperature by Athareh and Fatemeh [14] using weight loss, electrochemical impedance spectroscopy(EIS) and Tafel polarization measurements. The inhibition efficiencies obtained from all methods employed are in good agreement with each other. The obtained results show that compound (A) is a very good inhibitor with efficiency of $98 \%$ at $100 \mathrm{ppm}$ additive concentration in acid solution. Generally the inhibition efficiency increased with increase of inhibitor concentration. Changes in impedance parameters (Rct and Cdl) were indicative of adsorption of compound (A) on the metal surface, leading to the formation of a protective film. Tafel polarization measurements showed that the inhibitor (A) is a mixed type. The adsorption of the inhibitor on the surface of mild steel in $0.5 \mathrm{M}$ sulphuric acid was found to obey Langmuir's adsorption isotherm. Scanning electron microscopy (SEM) observation, confirmed the existence of an absorbed protective film on the metal surface.

\section{Material and Methods}

\subsection{Preparation of Sample and Solution}

The aluminium sample with a chemical composition of $99.500 \% \mathrm{Al}, 0.0024 \% \mathrm{Sn}, 0.0007 \% \mathrm{~Pb}$, $0.3202 \% \mathrm{Fe}, 0.0030 \% \mathrm{Mn}, 0.0015 \% \mathrm{Cu}, 0.0018 \% \mathrm{Mg}, 0.0074 \% \mathrm{Ti} 0.0045 \% \mathrm{Ni}, 0.0041 \mathrm{Cr}, 0.0060 \% \mathrm{Zn}$ and $0.0085 \% \mathrm{Si}$ was used in the present study. The metal sheet, was press cut in to $3 \times 2 \times 0.1 \mathrm{~cm}$ dimensions. The metal sheet was polish by emery paper of different grade. It was then degreased in ethanol, dried in acetone and kept in a desiccator to use during the experiment [15].

\subsection{Preparation of Solutions}

Phosphoric acid was used as corrosive media with percentage purity (85 \%) and density (1.685 g/L). The stock solution was used to prepare $1.4 \mathrm{M}$ concentration by diluting analytical grade of H3PO4 using double distilled water. Benzaldehyde (methoxy benzene) with percentage purity (95 \%) and density (1.04 g/L), thus, concentrations of the inhibitor used for the study ware 0.02, 0.04, 0.06, 0.08 and $0.1 \mathrm{M}$. Each of these 
concentrations was diluted in the prepared desired concentrations of acid for use as test solutions in weight loss experiment.

\subsection{Weight loss measurement}

uring weight loss experiment, the prepared aluminium samples were each suspended completely in $1.4 \mathrm{M}$ H3PO4 solution without and with different concentrations of benzaldehyde with the help of glass hooks at a temperature of $308 \mathrm{~K}$ and $3 \mathrm{hrs}$ immersion time. The volume of solution kept $100 \mathrm{~cm} 3$, the aluminium samples were retrieved after 3 hrs, washed by distilled water, dried well and reweighed. From the weight loss data, corrosion rate in $\mathrm{mg} / \mathrm{cm} 2$ was calculated.

\subsection{Temperature effect}

To study the effect of temperature on corrosion rate of aluminium, the prepared aluminium samples were completely immersed in $100 \mathrm{~cm} 3$ of $1.4 \mathrm{M}$ H3PO4 solution without and with different concentrations of benzaldehyde at 308, 313 and $318 \mathrm{~K}$ for 3 hrs.

\subsection{Fourier Transform Infrared Spectroscopic Analysis}

Fourier transform infrared spectroscopic analysis (FT-IR) analysis of the fresh inhibitor and that obtained from the corrosion product of aluminium immersed in in phosphoric acid solution in the presence of benzaldehyde inhibitor were carried out using Agilent Technology, FTIR (Cary 630) Fourier Transform Infrared Spectrophotometer. The samples were scan through $650-4000 \mathrm{~cm}-1$ wave number during the analysis.

\section{Results and Discussion}

\subsection{Weight loss experiments}

The weight loss and corrosion rate of aluminium in nitric acid solution in absence and presence of different inhibitor concentration $(0.02,0.04,0.06,0.08$ and $0.10 \mathrm{M})$ for an immersion time of 3 hrs was evaluated from the weight loss data by using the equation given below;

Weight Loss $=\mathrm{W}_{1}-\mathrm{W}_{2}$

Where $\mathrm{W}_{1}$ and $\mathrm{W}_{2}$ are the weight of coupons before and after immersion

$$
\text { Corrosion Rate }\left(\mathrm{mg} / \mathrm{cm}^{2} \mathrm{~h}^{-1}\right)=\frac{\text { Weight loss }(\mathrm{g})}{\text { Area }\left(\mathrm{cm}^{2}\right) \times \text { Time }(\mathrm{hrs})}
$$

The surface coverage $(\Theta)$ and inhibition efficiency (I.E) for different concentration of the inhibitor in acidic media have been evaluated from weight loss experiments using the equations below:

$$
\text { Surface coverage }=\frac{W_{u}-W_{i}}{W_{u}}
$$


Inhibition Efficiency $=\frac{W_{u}-W_{i}}{W_{u}} \times 100$

Where $\mathrm{W}_{\mathrm{u}}$ and $\mathrm{W}_{\mathrm{i}}$ are the weight of the coupon in the absence and presence of inhibitor.

Table 1. Variation of Corrosion Parameters for Corrosion of Aluminium in 1.4 M H3PO4 in the Absence and Presence of Different Concentrations of Inhibitor.

\begin{tabular}{|c|c|c|c|c|c|c|c|c|c|}
\hline \multirow{2}{*}{$\begin{array}{l}\text { Inhibitor } \\
\text { Conc. (M) }\end{array}$} & \multicolumn{3}{|c|}{ Corrosion rate $\left(\mathrm{mgcm}^{-2} \mathrm{~h}^{-1}\right)$} & \multicolumn{3}{|c|}{ Surface Coverage $(\theta)$} & \multicolumn{3}{|c|}{ Inhibition efficiency (\%) } \\
\hline & $308 \mathrm{~K}$ & $313 \mathrm{~K}$ & $318 \mathrm{~K}$ & $308 \mathrm{~K}$ & $313 \mathrm{~K}$ & $318 \mathrm{~K}$ & $308 \mathrm{~K}$ & $313 \mathrm{~K}$ & $318 \mathrm{~K}$ \\
\hline Blank & 1.5000 & 1.8332 & 2.2388 & - & - & - & - & - & - \\
\hline 0.02 & 0.8444 & 1.1888 & 1.5388 & 0.3889 & 0.3502 & 0.3121 & 38.88 & 35.02 & 31.21 \\
\hline 0.04 & 0.7833 & 1.0888 & 1.4388 & 0.4777 & 0.4052 & 0.3561 & 37.77 & 40.52 & 35.61 \\
\hline 0.06 & 0.7666 & 1.0388 & 1.3555 & 0.4889 & 0.4312 & 0.3942 & 48.89 & 43.12 & 39.42 \\
\hline 0.08 & 0.7611 & 0.9722 & 1.3166 & 0.4926 & 0.4690 & 0.4102 & 49.26 & 46.90 & 41.02 \\
\hline 0.10 & 0.7111 & 0.9277 & 1.2222 & 0.5259 & 0.4931 & 0.4535 & 52.59 & 49.31 & 45.35 \\
\hline
\end{tabular}

\subsection{Effect of inhibitor on corrosion rate and inhibition efficiency}

The result from Figure 1 and its presented in Table 1 shows the effect of inhibitor concentration on corrosion. The results shows that the corrosion rate decreases with increase in inhibitor concentration. Therefore the corrosion protection strengthened with increase in the concentration of inhibitor. This trend may be due to the fact that the adsorption and surface coverage increases with the increase in concentration of the inhibitor. Similar work was reported by Husaini et al. [16] who studied the corrosion inhibition of aluminium in nitric acid solution using anisaldehyde (4-methoxy benzaldehyde) as inhibitor and the result showed that corrosion rate decreases with increase in inhibitor concentration.

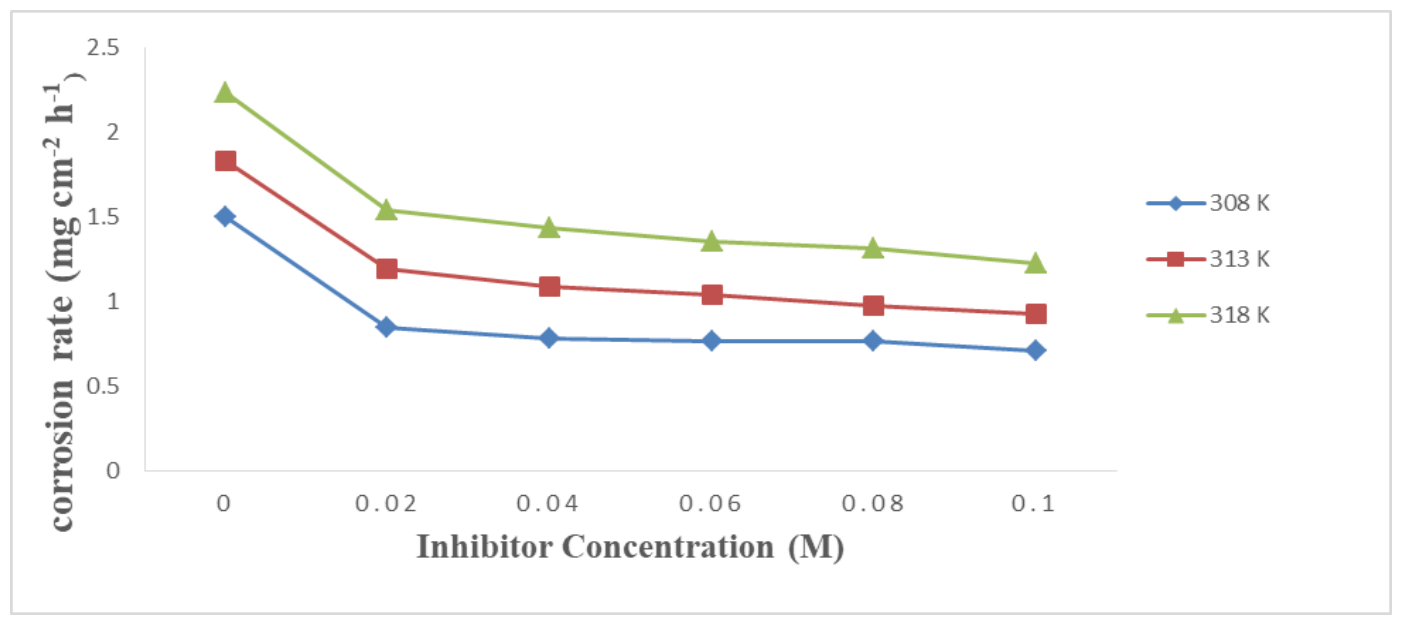

Fig. 1: Variation of Corrosion Rate with Inhibitor Concentration for $\mathrm{Al}$ Corrosion in $\mathrm{H}_{3} \mathrm{PO}_{4}$ 
Table 1 shows the values of surface coverage and inhibition efficiency increases with increase in inhibitor concentration. This effect of inhibition efficiency against different concentrations of the inhibitor is shown graphically in Figure 2. The highest inhibition efficiency value was obtained at highest inhibitor concentration. This is due to the increase of surface coverage of the inhibitor molecule on the surface of the aluminium, thus lead to the increase in protection of the aluminum against acid attack. Similar result was reported by Desai [12] during the investigation of aniline as corrosion inhibitor for aluminum in trichloroacetic acid and the values of surface coverage and inhibition efficiency was found to increase with increase in inhibitor concentration.

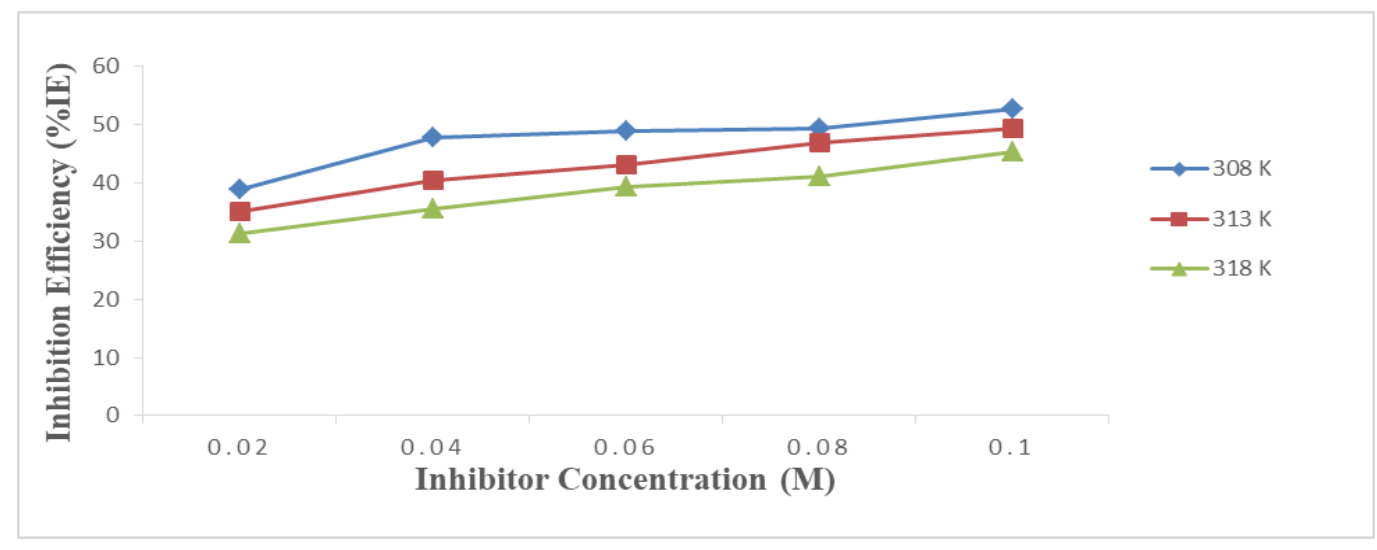

Fig. 2: Variation of Inhibition Efficiency with Inhibitor Concentration for $\mathrm{Al}$ Corrosion in $\mathrm{H}_{3} \mathrm{PO}_{4}$

\subsection{Effect of Temperature on Corrosion Rate and Inhibition Efficiency}

Figures 2 shows the variation of corrosion rate for the corrosion inhibition of aluminium in the presence and absence of inhibitor concentrations at different temperatures and its corresponding values presented in Table 1. The result showed that the corrosion rate increases with increase in temperature. The highest corrosion rate was obtained at highest temperature as can be seen from the result. This is because chemical reaction is usually increase with rise in temperature, due to the increase of the average kinetic energy of the reacting molecule. Similar work was reported by Yadav et al., [11] during the study of substituted amines as corrosion inhibitors for mild steel in $\mathrm{HCl}$ and observation depicts that rate of corrosion increases and no pitting was observed with increase in temperature.

Figures 4 shows the variation effect of temperature on the inhibition efficiency for the corrosion of aluminium in inhibitor concentrations at different temperatures. The result presented in Table 1 shows that the surface coverage and inhibition efficiency decreases with increase in temperature. Desorption and destabilization of the adsorbed inhibitor molecules on the metal surface occurs due to increase in temperature and thus lead to decrease in surface coverage and inhibition efficiency. Similar result was reported by Husaini and Ibrahim [17] during the thermodynamic and kinetic study on the corrosion of aluminium in hydrochloric acid using benzaldehyde as corrosion inhibitor and the result of inhibition efficiency was found to decrease with increase in temperature. 


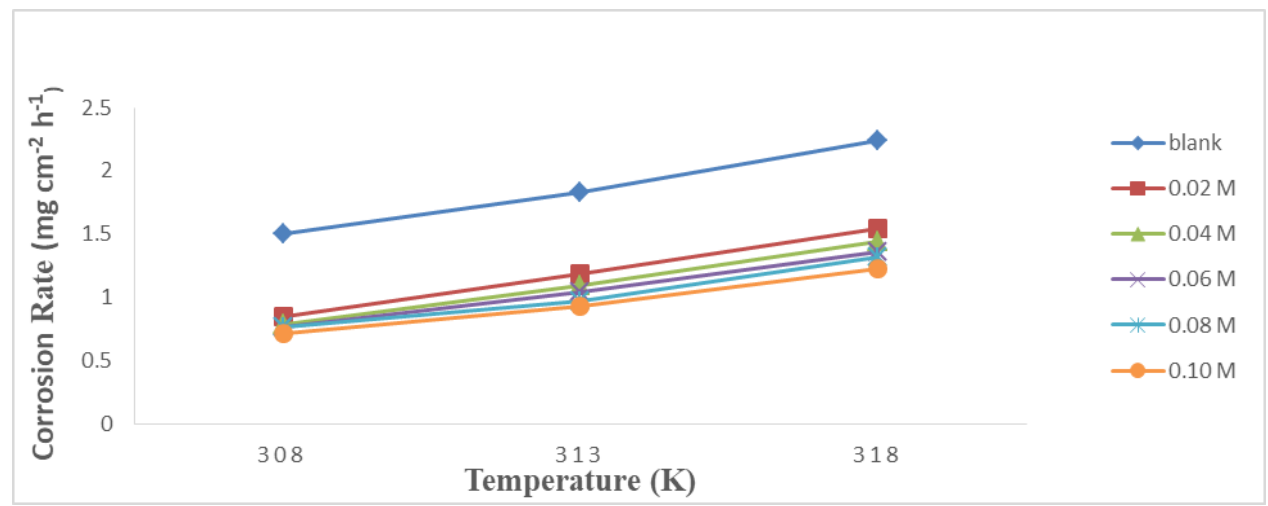

Fig. 3: Variation of Corrosion Rate with Temperature for $\mathrm{Al}$ Corrosion in $\mathrm{H}_{3} \mathrm{PO}_{4}$

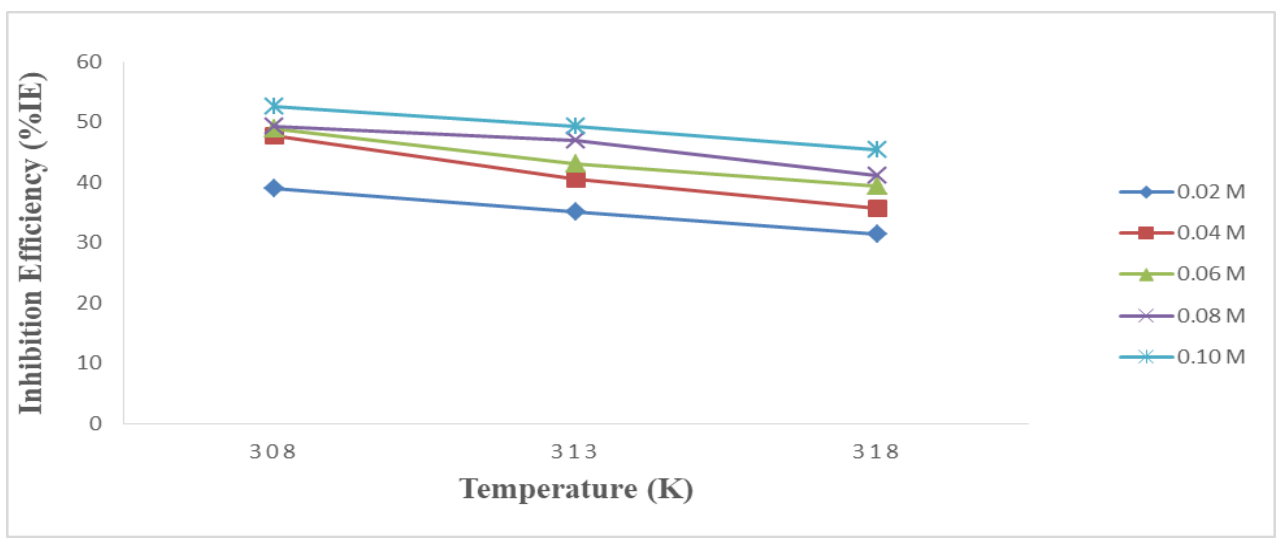

Fig. 4: Variation of Inhibition Efficiency with Temperature for $\mathrm{Al}$ Corrosion in $\mathrm{H}_{3} \mathrm{PO}_{4}$

\subsection{Kinetic Study}

\subsubsection{Activation Energy $\left(E_{a}\right)$}

Plotting of $\ln \rho$ (corrosion rate, $\mathrm{mg} \mathrm{cm}^{-2}$ ) against $1 / \mathrm{T}$ gave straight lines with slope equal to $-\mathrm{Ea} / \mathrm{R}$. The values of apparent activation energy for the aluminium dissolution process was evaluated by the equation given below

$$
\operatorname{Ln} \rho=\mathrm{B}-\frac{\mathrm{E}_{\mathrm{a}}}{\mathrm{RT}}
$$

Where $\rho$ is the rate of corrosion, Ea is activation energy, $\mathrm{T}$ is temperature and $\mathrm{R}$ is universal constant. Calculated activation energy values are given in table 2. As can be seen from the Table, activation energy values strongly increase in the presence of inhibitors. This trend indicates that the adsorption of the inhibitor molecules occurs through the mechanism of physical adsorption as reported by Musa et al. [18]. The observed trend from the data is consistent with investigations reported by Patel et al. [19] during electrochemical impedance study on the corrosion of pure aluminium in hydrochloric acid solution using schiff bases and the 
result for the activation energy in uninhibited solution was $25.9 \mathrm{kJmol}^{-1}$ while in the presence of Schiff base 1 and 2 were 79.5 and $85.8 \mathrm{kJmol}^{-1}$.

\subsubsection{Rate Constant (k)}

Corrosion process is heterogeneous that composed of cathodic and anodic reactions with different or equal rate. This made kinetic analysis of the data necessary. In this research $\mathrm{Wi}$, is considered as the initial weight of aluminum at time $\mathrm{t}, \Delta \mathrm{W}$ is the weight loss, $(\mathrm{Wi}-\Delta \mathrm{W})$ is the weight change at time $\mathrm{t}$ and first order rate constant is $\mathrm{k}$.

$$
\ln (\mathrm{Wi}-\Delta \mathrm{W})=-\mathrm{k}_{1} \mathrm{t}+\ln \Delta \mathrm{W}
$$

From equation 6 , the plots of $\ln (\mathrm{Wi}-\Delta \mathrm{W})$ versus time at $308 \mathrm{~K}$ gave a linear variation which confirms a first order reaction with respect to the aluminium corrosion in H3PO4 and in the presence of inhibitor. The rate constant for the first order reaction calculated from the slope of the plot were presented in Table 2. From the result the values of rate constant $(\mathrm{k} 1)$ for the corrosion of aluminium in uninhibited acid solution was found to be higher than that of inhibited acid solution. This confirmed the inhibition action of the inhibitor in $\mathrm{H}_{3} \mathrm{PO}_{4}$ solution.

\subsubsection{Half Life $\left(t_{1 / 2}\right)$}

The half-life $\left(\mathrm{t}_{1 / 2}\right)$ was calculated from equation 7

$$
\mathrm{t}_{1 / 2}=\frac{0.693}{k}
$$

The values of the half-live $\left(\mathrm{t}_{1 / 2}\right)$ presented in Table 2 increased from uninhibited acid solution to inhibited solution. The higher values of half-live in the presence of inhibitor compared to the uninhibited acid solution support the earlier results that corrosion rate decreases in the presence of the inhibitor.

Table 2. Kinetic Parameters for Aluminium Corrosion with and without various inhibitor concentrations

\begin{tabular}{cccc}
\hline $\begin{array}{c}\text { Inhibitor } \\
\text { Concentration (M) }\end{array}$ & $\begin{array}{c}\text { Activation Energy } \\
\left(\mathbf{k J ~ m o l}^{\mathbf{- 1}}\right)\end{array}$ & Rate Const. $\left.\mathbf{( k \times \mathbf { 1 0 } ^ { - \mathbf { 3 } } ) \mathbf { ( h o u r }} \mathbf{- 1}^{\mathbf{-}}\right)$ & Half-life (hours) \\
\hline Blank & 32.61 & 9.12 & 075.94 \\
0.02 & 44.09 & 5.54 & 124.93 \\
0.04 & 44.60 & 5.03 & 137.55 \\
0.06 & 46.42 & 4.73 & 146.38 \\
0.08 & 48.90 & 4.70 & 147.44 \\
0.10 & 49.53 & 4.56 & 151.80 \\
\hline
\end{tabular}

\subsection{Thermodynamic Study}

Enthalpy change $(\Delta \mathrm{H})$ and entropy change $(\Delta \mathrm{S})$ of the corrosion process were obtained by using the linear form of transition state equation.

$$
\ln \left(\frac{\mathrm{C}_{\mathrm{R}}}{\mathrm{T}}\right)=\ln \left(\frac{\mathrm{R}}{\mathrm{Nh}}\right)+\left(\frac{\Delta \mathrm{S}}{R}\right)-\left(\frac{\Delta \mathrm{H}}{R T}\right)
$$


Where $\mathrm{h}$ is the Planck's constant, $\mathrm{N}$ is the Avogadro's number, $\mathrm{R}$ is the universal gas constant and $\mathrm{T}$ is the absolute temperature. Straight line was obtained from the plots of $\ln \left(C_{R} / T\right)$ versus $1 / T$ with a slope equal to $-\Delta H / R$ and intercept equal to $\ln (\mathrm{R} / \mathrm{Nh})+\Delta \mathrm{S} / \mathrm{R}$ from which the values of $\Delta \mathrm{H}$ and $\Delta \mathrm{S}$ were evaluated. From the result presented in Table 2, the positive values of $\Delta \mathrm{H}$ indicate the endothermic nature of dissolution the dissolution process, and thus suggests the slower dissolution of aluminium in the presence of inhibitor. The sign of $\Delta S$ is negative in both uninhibited and in the presence of inhibitor. Therefore, the negative sign of entropy is attributed to the increase in solvent entropy and to more positive water desorption enthalpy.

Table 3. Enthalpy change $(\Delta \mathrm{H})$ and Entropy change $(\Delta \mathrm{S})$ of the reaction process with various concentrations of the inhibitor

\begin{tabular}{ccc}
\hline Inhibitor Concentration $(\mathbf{M})$ & $\mathbf{\Delta} \mathbf{H}\left(\mathbf{k J ~ m o l}^{\mathbf{- 1}}\right)$ & $-\boldsymbol{\Delta S}\left(\mathbf{k J ~ m o l}^{\mathbf{1}} \mathbf{k}^{\mathbf{- 1}}\right)$ \\
\hline blank & 30.01 & 201.81 \\
0.02 & 41.49 & 170.72 \\
0.04 & 41.99 & 168.61 \\
0.06 & 43.81 & 162.51 \\
0.08 & 46.29 & 153.59 \\
0.10 & 46.93 & 152.20 \\
\hline
\end{tabular}

\subsection{Adsorption Consideration}

Adsorption isotherms gives an important information about the mechanism of interaction between the inhibitor molecules and the metal surface. In this view the values of the degree of surface coverage were obtained from weight loss measurements using the equation 3. Attempts were made to fit the values in to different adsorption isotherms and the values of correlation coefficient $\left(\mathrm{R}^{2}\right)$ were used to determine the best fit isotherm. Langmuir adsorption isotherm was by far found to give best result among other tested isotherm, which has the form:

$$
\frac{C}{\theta}=\frac{1}{\mathrm{~K}_{\mathrm{ads}}}+\mathrm{C}
$$

Where $\Theta$ is the degree of surface coverage, $C$ is the concentration of the inhibitor and $\mathrm{K}_{\mathrm{ads}}$ is adsorption equilibrium constant of adsorption process. The plot of $\mathrm{C} / \Theta$ against inhibitor concentration gave a straight line with slope and $\mathrm{R}^{2}$ close to unity indicating that the data obtained from the experiment fit well into the Langmuir adsorption isotherm. The values of $\mathrm{K}_{\text {ads }}$ presented in Table 4 were found to decrease with increase in temperature suggesting that the molecules of the inhibitor were physically adsorbed on the aluminium surface. Similar result was reported by Husaini et al. [20].

Gibbs free energy of adsorption $\left(\Delta \mathrm{G}_{\mathrm{ads}}\right)$ and adsorption equilibrium constant $\left(\mathrm{K}_{\mathrm{ads}}\right)$ are related by the equation given below

$$
\Delta \mathrm{G}_{\mathrm{ads}}=-\mathrm{RT} \ln \left(55.5 \times \mathrm{K}_{\mathrm{ads}}\right)
$$

The calculated values of Gibbs free energy of adsorption for the inhibitor at a given temperature of 308, 313 and $318 \mathrm{~K}$, are shown in Table 4. The negative values is an indication of spontaneous adsorption of inhibitor molecules on the metal surface. The obtained results indicated that the inhibitor was physically adsorbed on the surface of the metal since all the values of $\Delta \mathrm{G}_{\text {ads }}$ are around $-20 \mathrm{kJmol}^{-1}$. Generally values of $\Delta \mathrm{G}_{\mathrm{ads}}$ below or aound $-20 \mathrm{kJmol}^{-1}$ are associated with electrostatic interaction (physisorption) between charged inhibitor molecules and a charged metal surface. While the values of $\Delta \mathrm{G}_{\text {ads }}$ at or more negative than 
$-40 \mathrm{kJmol}^{-1}$ are consistent with charge sharing or charge transfer from the inhibitor molecules to the surface of the metal to form a co-ordinate type of bond (chemisorption).

Table 4. Adsorption Parameters Deduced from Langmuir Adsorption Isotherm for Corrosion Inhibition of Aluminium.

\begin{tabular}{llll}
\hline Temperature (K) & $\mathbf{R}^{\mathbf{2}}$ & $\mathbf{K}_{\text {ads }}$ & $\Delta \mathbf{G}_{\text {ads }}\left(\mathbf{k J ~ \mathbf { ~ m o l } ^ { \mathbf { - 1 } } )}\right.$ \\
\hline 308 & 0.997 & 45.35 & -20.05 \\
313 & 0.996 & 41.48 & -20.15 \\
318 & 0.990 & 38.44 & -20.27 \\
\hline
\end{tabular}

\subsection{Infrared Spectroscopic Analysis Results}

Fourier transform infrared spectroscopic analysis of the metal, organic compound and that of the corrosion product are shown in Figures 5 and 6. Figure 5 is the result for the FT-IR analysis of the organic compound which shows the presence of $\mathrm{C}=\mathrm{O}$ stretch of carbonyl group, $\mathrm{C}-\mathrm{H}$ stretch of aldehyde and $\mathrm{C}-\mathrm{H}$ stretch of aromatic. Figure 6 shows the result for the FT-IR analysis of the corrosion product. The presence of $\mathrm{C}=\mathrm{O}$ stretch of carbonyl group, C-H stretch of aldehyde and C-H stretch of aromatic in the FT-IR result of the corrosion product indicated that there is the formation of the thin layer of the inhibitor molecules on the surface of the aluminium.

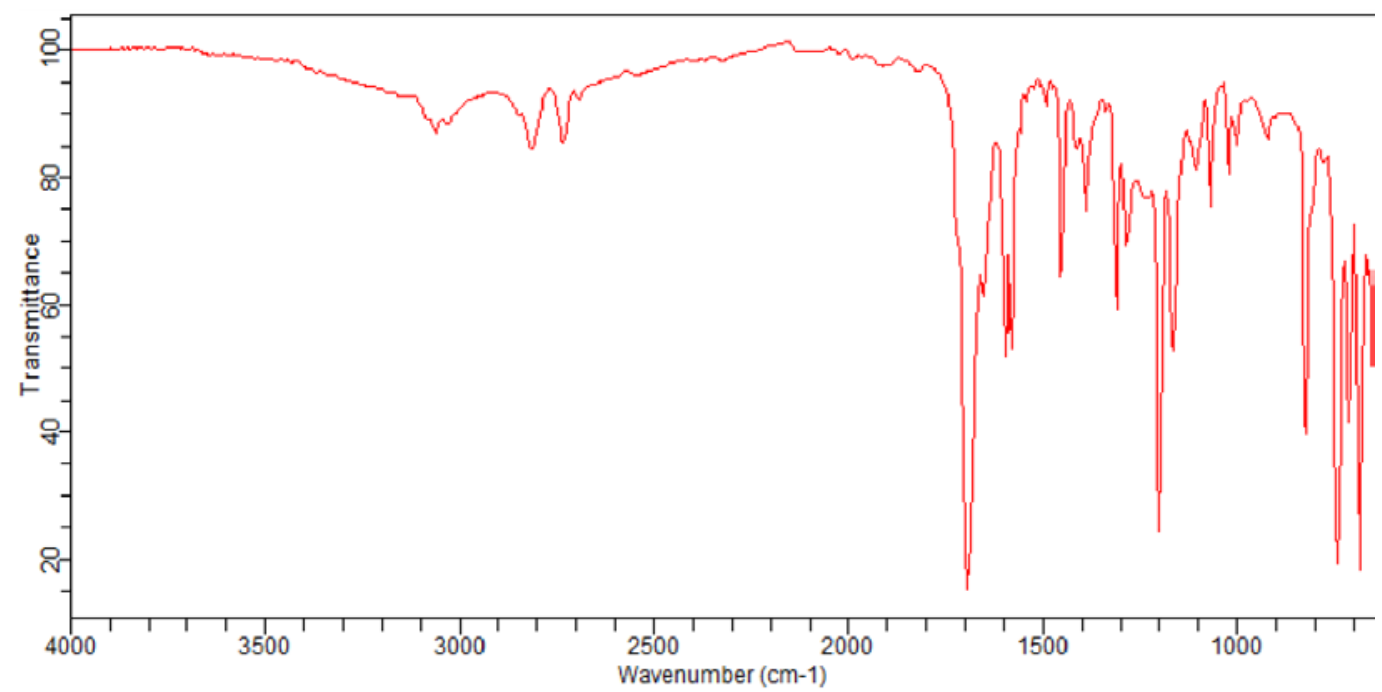

Fig.5. FT-IR Spectra of Organic Compound 


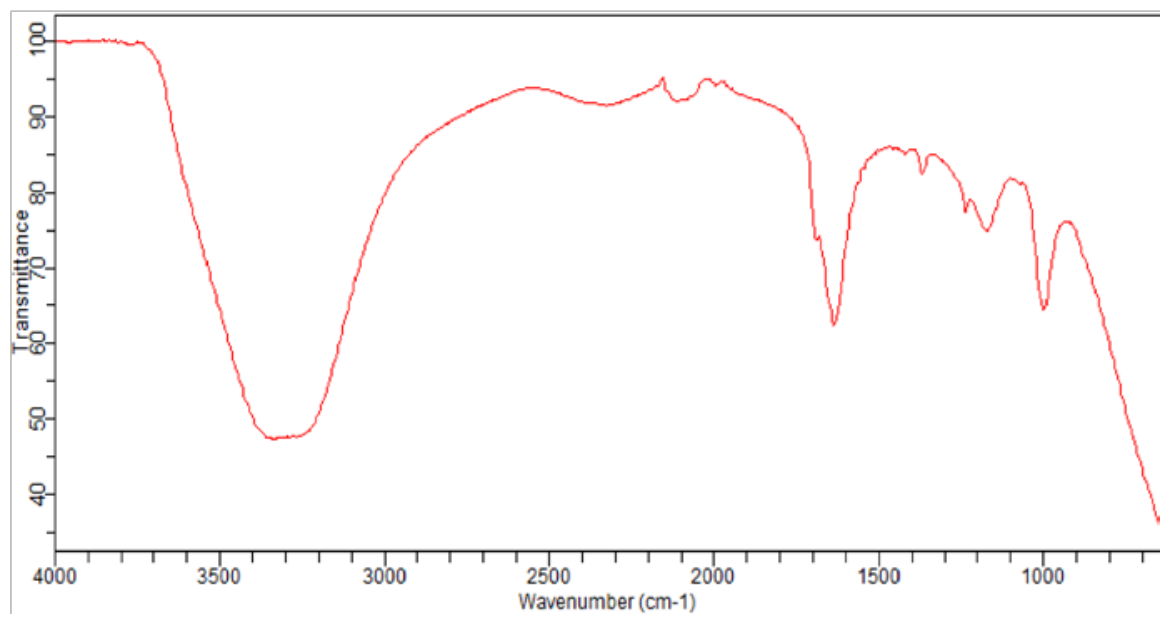

Fig.6. FT-IR Spectra of Corrosion Product

\section{CONCLUSION}

The study revealed that the organic compound (benzaldehyde) served as a potential eco-friendly corrosion inhibitor for the corrosion of aluminium in phosphoric acid solution. The use of this investigated compound will reduce the rates of usage of toxic inhibitors that are harmful to environment. This research is in advancement of contribution toward the sustained world-wide in looking for friendly, non-toxic and commercially available corrosion inhibitors. It can be concluded that increase in inhibitor concentration caused the increase in inhibition efficiency but inhibition efficiency decreased with rise in temperature. The values of enthalpy change were positive, which is an indicative of endothermic reaction. The negative values of Gibbs free energy of adsorption shows that the corrosion process was spontaneous and adsorption took place through the mechanism of physical adsorption. The result of Fourier transform infrared spectroscopic analysis of the corrosion product shows that there is the formation of thin layer of the inhibitor on the aluminium surface, due to the appearance of functional group of the inhibitor on the corrosion product. The adsorption of the inhibitor molecule on the aluminium was found to accord with Langmuir adsorption isotherm.

The current research was only limited to weight loss method, so it does not include other method of determining the corrosion process, such as electrochemical method. So it's recommended that the feature study should include other method such as potentiodynamic polarization method and titrimetric method.

\section{References}

[1] Paulina, A. L., Octavio, O. X., Diego, G. L., Natalya, V. L., Marco, A. D., Irina, V. L. (2014). The Inhibition of Aluminum Corrosion in Sulphuric Acid by Poly (1-Vinyl-3-alkyl- Imidazlium Hexafluoro-Phosphate). Journal of Materials. 7: 5711- 5734.

[2] Amin, M.A., Mohsen, Q. and Hazzai. O.A. (2009). Synergistic effect of I- ions on the corrosion inhibition of $\mathrm{Al}$ in $1.0 \mathrm{M}$ phosphoric acid solution by purine. Mater. Chem. Phys. 114: 908-914.

[3] Benabdellah, M. Benkaddour, M. Hammouti, B. Bendahhou, M. Aouniti, A. (2006). Inhibition of steel corrosion in 2M H3PO4 by Artemisia oil. Appl. Surf. Sci. 252 (18): 6212-6217. 
[4] Noor, E. (2005). The inhibition effect of mild steel corrosion in phosphoric acid solutions by some $\mathrm{N}$-heterocyclic compounds in the salt form. Corros. Sci. 47(1): 33

[5] Wang, (2006). Inhibition of mild steel corrosion in phosphoric acid solution by triazolederivatives. Corros. Sci. 48: 608-616

[6] Upadhyay, R.K and Mathur, S.P. (2007). Effects of Schiff's bases as corrosion inhibitors on mild steel in sulphuric acid. E-Journal of chemistry. 4(3):408-414.

[7] Ashish, K. S. and Quraishi, M. A. (2012). Study of Some Bidentate Schiff Bases of Isatin as Corrosion Inhibitors for Mild Steel in Hydrochloric Acid Solution. Internotional Journal of Electrochemical Science. 7: 3222-3241.

[8] Hachelef, H., Benmoussat, A,. Khelifa, A., Athmani, D. and Bouchareb, D. (2016): Study of corrosion inhibition by Electrochemical Impedance Spectroscopy method of 5083 aluminum alloy in $1 \mathrm{M} \mathrm{HCl}$ solution containing propolis extract. J. Mater. Environ. Sci. 7: 1751-1758

[9] Zhang, Q. B. and Hua, Y. X. (2010). Corrosion inhibition of aluminum in hydrochloric acid solution by alkylimidazolium ionic liquids. Mater. Chem. Phys. 119: 57-64.

[10] Megalai, M.S., Ramesh, R. and Maniula, P. (2013). Inhibition of Corrosion Mild Steel in Acid Media by Trazodone Drug. Research Desk. 2(4): 326-333.

[11] Yadav, M., Kumar, S., Sharma, U. and Yadav, P.N. (2013). Substituted amines as corrosion inhibitors for N80 steel in 15\%HCl. Journal of Material and Environmental Science. 4(5): 691-700.

[12] Desai, P. S. (2015). Aniline as Corrosion Inhibitor for Aluminum in Trichloroacetic Acid. European Journal of Pharmaceutical and Medical Research. 2(2): 527-537.

[13] Arvnabh, M., Godhani, D.R. And Anil S. (2011). Diamines as corrosion inhibitors for aluminium alloy in organic acid. Asian Journal of Experimental Chemistry. 6(1): 38-41

[14] Athareh, D. and Fatemeh, B. (2011). A New Synthesized Corrosion Inhibitor for Mild Steel in $0.5 \mathrm{M}$ H2SO4. Gazi University Journal of Science. 24(2): 219-226.

[15]. Husaini, M., Usman, B. and Ibrahim, M. B. (2018). Evaluation of corrosion behavior of aluminum in different environment. Bayero Journal of Pure and Applied Sciences. 11(1): 88-92.

[16] Husaini, M., Usman, B. and Ibrahim, M. B. (2019). Study of corrosion inhibition of aluminium in nitric acid solution using Anisaldehyde (4 - methoxy benzaldehyde) as inhibitor. Algerian Journal of Engineering and Technology.

[17] Husaini, M and Ibrahim, M.B (2019). Thermodynamic and Kinetic study on the corrosion of aluminium in hydrochloric acid using benzaldehyde as corrosion inhibitor. International Journal of Engineering and Manufacturing. 9(6): 53-64

[18] Husaini, M., Usman, B., Ibrahim, M. B. (2020). Effect of Aniline as Corrosion Inhibitor on the Corrosion of Aluminium in Hydrochloric Acid Solution. Research Journal of Chemistry and Environment. 24(2): $1-8$.

[19] Patel, A. S., Panchal, V. A., and Shah, N. K. (2012). Electrochemical Impedance Study on the Corrosion of Al-Pure in Hydrochloric Acid Solution Using Schiff bases. Indian Academy of Sciences. 35(2): 283-290.

[20] Husaini, M., Usman, B., Ibrahim, M. B. (2019). Inhibitive Effect of Glutaraldehyde on the Corrosion of Aluminium in Hydrochloric Acid Solution. Journal of Science and Technology. 11(2): 8-16 


\section{Authors' Profiles}

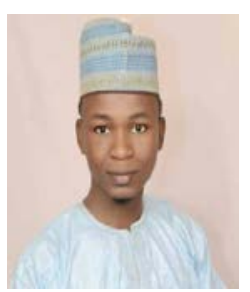

Musa Husaini, B.Sc Chemistry (Umaru Musa Yaradua University Katsina, Nigeria); M.Sc Physical Chemistry (Bayero University Kano, Nigeria); Ph.D. Research in Physical Chemistry is in progress at Bayero University Kano. Currently working on corrosion protection of metallic substances.

How to cite this paper: Musa Husaini, Muhammad Bashir Ibrahim. "Investigation of Inhibition Potential Effect of Organic Compound for the Corrosivity of Phosphoric Acid on Aluminium ", International Journal of Engineering and Manufacturing(IJEM), Vol.10, No.1, pp.41-53, 2020. DOI: 10.5815/ijem.2020.01.04 